\title{
La transición a la universidad desde el bachillerato y desde el CFGS. La importancia de los factores socioeconómicos
}

\author{
Lidia Daza Pérez
}

Universidad de Barcelona. Departamento de Sociología

ldaza@ub.edu

ORCID: 0000-0003-4307-7625

\section{Helena Troiano}

Universidad Autónoma de Barcelona. Departamento de Sociología helena.troiano@uab.cat

ORCID: 0000-0002-8352-2415

\section{Marina Elias Andreu}

Universidad de Barcelona. Departamento de Sociología

marinaelias@ub.edu

ORCID: 0000-0002-8268-4965

Recepción: 05-02-2018

Aceptación: 24-06-2018

Publicación: 19-02-2019

\section{Resumen}

El momento de transición de la educación postobligatoria a la educación superior es un proceso de decisión que acumula las desigualdades que se han ido produciendo en cruces anteriores del sistema, como también los efectos que el rendimiento académico ejerce a lo largo de los estudios. Ante estas evidencias, el alcance de la expansión educativa en términos de la educación superior, en paralelo al diseño de diferentes itinerarios que permiten un acceso sin cuotas a la universidad, concretamente para los estudiantes procedentes de ciclos formativos de grado superior, plantea nuevas preguntas acerca de la persistencia o no de esta desigualdad de oportunidades educativas.

Para intentar responder a esta cuestión, el artículo analiza el hecho de transitar a la universidad diferenciando entre aquellos estudiantes que poseen el título de bachillerato y los que obtienen el de ciclo formativo de grado superior (CFGS). Mediante la Enquesta a la Joventut de Catalunya se realizan tablas de contingencia y una regresión logística binaria para comprobar la probabilidad de éxito (ir a la universidad) en los dos colectivos, de acuerdo con diferentes variables propuestas para el modelo.

Los resultados muestran una incidencia relevante de las notas para los estudiantes de orígenes sociales más bajos. En cambio, más que una opción, para los jóvenes pertenecientes a familias con más recursos socioeconómicos ir a la universidad es su trayectoria natural, independientemente de las notas (efecto compensación). Se detectan también claras dife- 
rencias entre los alumnos de bachillerato, que transitan a la universidad en proporciones elevadas, y los de CFGS, que lo hacen en proporciones menores.

Palabras clave: itinerario educativo; desigualdad social; efectos secundarios; nivel educativo familiar; ocupación; notas

Abstract. Transition to university from high school and second-cycle vocational training: The importance of socioeconomic factors

The transition from upper secondary education to tertiary education accumulates the inequalities produced in previous crossings of the educational system and is also influenced by academic performance. Moreover, educational expansion, in addition to widening possible schooling tracks for accessing university, poses new questions about the persistence of this inequality of educational opportunities.

To try to answer this question, this article analyses the demand for higher education, differentiating between students with an upper secondary school diploma (academic track) and those with a second-cycle vocational training certificate. Using data from the Joventut de Catalunya Survey, contingency tables are constructed and binary logistic regression is performed to test the probability of success (going to university) according to different model variables.

The results show that grades have an important influence on students with a lower socioeconomic status. For students with more socioeconomic resources, going to university is a natural path rather than an option, regardless of grades (compensation effect). There are also clear differences between upper secondary school graduates, who enrol in university in high proportions, and those from second-level vocational training programmes, who do so in smaller proportions.

Keywords: educational pathway; social inequality; secondary effects; family education level; occupation; grades

\section{Sumario}

\section{Introducción 6. Resultados}

2. La transición a la universidad 7. Conclusiones

3. Efectos primarios y efectos secundarios Referencias bibliográficas

4. Modelo de análisis Anexo

5. Datos, variables y técnicas de análisis

\section{Introducción}

En las últimas décadas se ha evidenciado una expansión del sistema educativo español y, en concreto, un mayor acceso de jóvenes a la educación universitaria. Según Carabaña (2015), la tasa bruta de escolaridad universitaria subió del $30 \%$ al 45\% entre 1990 y 2000, casi se estancó entre 2000 y 2007 y ha vuelto a crecer hasta un $55 \%$ entre 2007 y 2011 . Eso ha comportado que jóvenes con perfiles cada vez más heterogéneos realicen estudios superiores. Según datos de la OCDE (2013), España vive un cambio de tendencia en la proporción de 
jóvenes escolarizados en la educación secundaria superior. En la mayoría de los países, este segmento tiene una amplitud similar o superior al de la educación terciaria, pero aquí la mayoría de jóvenes que prosiguen con esta etapa lo hacen a través del bachillerato, un nivel pensado como de transición a la educación terciaria, mientras que la opción de los ciclos formativos resulta más minoritaria, pese a dotar de un título con cualificación para la inserción laboral de los jóvenes. No obstante, en los últimos años se ha incrementado la proporción de personas escolarizadas en la segunda etapa de la educación secundaria. Por otro lado, en el año 2010 (Real Decreto 558/2010) se produce un cambio de normativa que implica una modificación del volumen de estudiantes que acceden a la universidad por la vía de los ciclos formativos de grado superior (CFGS). Deja de existir una cuota de acceso para este colectivo, con lo que aumentan sus oportunidades de matriculación directa a la educación superior, con la opción de subir nota mediante las pruebas específicas. Esto nos lleva a explorar cómo, en un contexto de ampliación del acceso a la universidad y de apertura a los CFGS, el origen social afecta a la entrada de los jóvenes a la universidad desde la vía tradicional (secundaria académica) y desde la vía ampliada (secundaria profesional) (Shavit y Blossfeld, 1993; Breen et al., 2009).

El trabajo que aquí se presenta analiza la influencia del origen social familiar de los estudiantes catalanes que transitan hacia la universidad. Concretamente, se trata de jóvenes que para el año 2012 estaban en posesión del título requerido para poder tomar la decisión sobre si acceder o no a la universidad, puesto que habían terminado el bachillerato o bien los estudios de técnico superior de formación profesional. Se examina la influencia que tiene la clase social en la decisión de ir a la universidad, controlada por el efecto del rendimiento previo. Lo novedoso de este trabajo radica en el hecho de analizar la transición a la educación superior en referencia a dos perfiles de estudiante claramente diferenciados, como veremos más adelante: los alumnos que han cursado la vía postobligatoria más tradicional habiendo pasado por el bachillerato y los que han seguido un itinerario más largo, puesto que han pasado por un CFGS. Existen diversos trabajos sobre los determinantes del tránsito de la secundaria obligatoria a la secundaria postobligatoria (Bernardi y Cebolla, 2014; Bernardi y Requena, 2010), pero son muchos menos los realizados sobre el tránsito a la universidad por parte de los jóvenes que han finalizado una educación postobligatoria. Un ejemplo de ello es el trabajo de Gil Izquierdo et al. (2010), aunque sus autores recogen de forma indistinta a todos los individuos que han finalizado la educación postobligatoria (bachillerato o ciclo formativo de grado medio), sin diferenciar la vía de entrada. Algunos de los motivos que pueden explicar esta falta de conocimiento sobre este punto de inflexión educativa y su emergencia como objeto de análisis sociológico pueden atribuirse, por un lado, al hecho de que en España no se dispone de los datos necesarios que permitan realizar este tipo de análisis y, por otro, que no ha sido hasta el año 2010 que la presencia de los estudiantes procedentes de un CFGS ha aumentado lo suficiente como para ser objeto de análisis, debido al cambio en la política de acceso a la universidad. 
El artículo se organiza de manera que en un primer apartado se analiza el perfil diferencial de estos dos grupos de jóvenes con opciones reales de acceder a la universidad: el colectivo de estudiantes que han obtenido el título de bachillerato y aquel con el título de CFGS. Cabe recordar que, según el sistema educativo actual, el acceso al CFGS se puede hacer desde el mismo bachillerato o desde los ciclos formativos de grado medio (CFGM). A continuación, en un segundo apartado, examinamos cuáles son los principales predictores de realizar la transición a la universidad en cada caso, centrándonos sobre todo en el rendimiento previo y en el origen social, además de incorporar la interacción entre las dos variables.

\section{La transición a la universidad}

El momento de realizar la transición de la educación postobligatoria a la superior es un proceso de decisión que acumula las desigualdades que se han ido produciendo en cruces anteriores del sistema, como también los efectos que el rendimiento educativo ejerce a lo largo de los estudios. Por este motivo, es particularmente importante a este nivel adoptar la separación clásica entre lo que se denomina los efectos primarios (EP en adelante) y los efectos secundarios (ES en adelante), que popularizó Boudon (1974) en la versión americana de L'inegalité des chances (1973), y que hizo fortuna gracias a la contribución de un buen número de autores posteriores (Breen y Goldthorpe, 1997; Mayer et al., 2007; Breen et al., 2009; Jackson, 2013).

Los EP explican la desigualdad educativa a partir de cómo el origen social influye en la adquisición de competencias académicas a través de diferentes mecanismos como, por ejemplo, la disponibilidad de recursos económicos, culturales y sociales de las familias, y que ponen a disposición de su descendencia. Esto tiene una relación directa con el rendimiento de sus hijos. Los ES, en cambio, explican cómo el origen social afecta directamente a las decisiones educativas, a pesar de que los hijos de una y otra clase social tengan el mismo nivel de competencias. Es decir, alumnos con las mismas notas toman decisiones diferentes respecto a qué itinerario educativo seguir de acuerdo con su origen social. Eso ocurre porque entra en juego, desde la perspectiva de la elección racional, la valoración de coste-beneficio que realizan los estudiantes acerca de su posible decisión, así como las expectativas que tienen respecto a los estudios, y ambos elementos están estrechamente relacionados con su posición en la estructura social. Se trata, por tanto, de comprender en qué medida la desigualdad referida a las oportunidades educativas es atribuible al desempeño del estudiante o bien a las decisiones que toman en cada encrucijada educativa en función de la clase social de origen. Igual que hicieran Carabaña y De la Fuente (2016) al analizar las condiciones de empleo de los licenciados en Ciencias Sociales y Humanidades, se pretende comprobar si persiste clasismo, en su caso en el mercado de trabajo, en nuestro caso en la universidad, y cómo se evidencia en cada uno de los colectivos analizados. 


\section{Efectos primarios y efectos secundarios}

Desde el punto de vista de los EP, es habitual constatar la relación entre variables que se refieren a la clase social de la familia - tanto si se utilizan los ingresos como la ocupación, la relación con la propiedad o el nivel académico de los progenitores - y el rendimiento que obtienen sus hijos en la escuela.

Pero si hablamos de los ES, deben contemplarse dos tipos de mecanismos: el efecto que la clase social ejerce en la elección de continuar estudiando (Bernardi y Requena, 2010) y este mismo mecanismo a la hora de tomar un camino u otro intermediado por las notas (Jackson, 2013; Bernardi y Cebolla, 2014). De modo que, si bien las notas son el resultado de los EP, también influyen en los ES como suministradores de oportunidades de elección (las notas obtenidas pueden abrir o cerrar puertas de continuidad de estudios a diferentes vías), y como información sobre la probabilidad futura de éxito, de la cual el individuo hace una estimación a partir de la valoración de su propia capacidad (ver figura 1).

Tanto el sexo como las notas previas son variables que normalmente sirven de control de los ES. En el mismo sentido, se utiliza otro conjunto de variables que, más o menos directamente, se relacionan con el coste de continuar estudiando (Gil Izquierdo et al., 2010; Torrents, 2015): ocupación de los progenitores, renta del hogar, tamaño del hogar, presencia de menores, proporción de desocupados, densidad de población (vinculado a la lejanía de la oferta de educación superior), etc. Gil Izquierdo et al. (2010) repasan las aportaciones de diversas investigaciones en España y llegan a la conclusión de que el peso de estas razones económicas para decidir la continuación de estudios hacia la universidad se ha ido reduciendo a lo largo de las décadas de 1980, 1990 y 2000. El retorno esperado según la opción escogida, en cambio, se introduce raramente en los análisis.

El último factor que casi siempre se introduce en los análisis de los ES es el del capital cultural familiar. Lo más habitual es utilizar el indicador del nivel

Figura 1. Los efectos primarios y los efectos secundarios en el acceso a la universidad

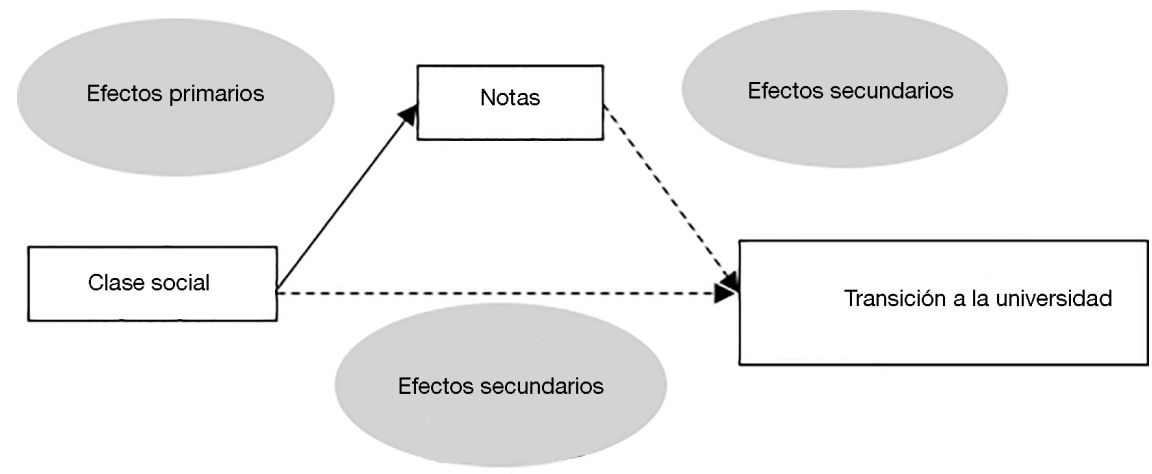

Fuente: elaboración propia a partir de Jackson (2013). 
de estudios de los progenitores (Marqués-Perales y Gil-Hernández, 2015). En la explicación sobre los ES algunos autores introducen, a través de este tipo de variables, conceptos próximos al habitus, sobre todo por el mecanismo de la tendencia inercial hacia los estudios (o no) en función del capital cultural familiar (Hatcher, 1998; Becker y Hecken, 2009). También se explica su influencia a través del efecto información, según el cual un buen conocimiento del sistema educativo por parte de los adultos de la familia permitiría valorar más adecuadamente las vías posibles, los retornos probables y la dificultad real de compleción de unos estudios.

Bernardi y Cebolla (2014) identifican dos tipos de aproximaciones al análisis de los EP y ES, utilizando también técnicas de análisis diferenciadas, que consideran que tales efectos actúan de forma aditiva y sin tener en cuenta que puede haber desigualdades en la propensión a hacer una transición o no dependiendo de las notas obtenidas en la etapa previa. Dichos autores rebaten este supuesto poniendo de manifiesto cómo en realidad se trata de efectos que interaccionan. Es decir, el impacto desigualador de la familia de origen puede ser mayor o menor en función del rendimiento previo del individuo. En concreto, hablan de un efecto de compensación que supone que la desigualdad entre clases sociales es mayor entre los alumnos que obtienen peores notas (Bernardi, 2012). Por tanto, los peores estudiantes de clase alta tienden a transitar en mayor medida que los malos estudiantes de clase trabajadora. Dicho de otro modo, ante las malas notas de los hijos, las clases altas disponen de mayores recursos (económicos, culturales y sociales) para compensar este bajo rendimiento académico $y$, por tanto, pueden invertir en su proceso formativo para minimizar el riesgo de fracaso. Otra forma de desigualdad que exponen, aunque no es el propósito de este trabajo adentrarse en ello pero sí puede ser objeto de otra investigación, es la debida al efecto información incompleta. Se trata del fenómeno consistente en el hecho de que las mayores desigualdades se producen entre clases sociales cuando las notas son intermedias y, por tanto, no existe una interpretación clara sobre la posibilidad de éxito o de fracaso al seguir estudiando (Bernardi y Cebolla, 2014).

\section{Modelo de análisis}

La aproximación que realizamos para identificar los determinantes de la transición a la educación superior se enmarca en la perspectiva de la elección racional, adoptando la formalización de Breen y Goldhorpe (1997) para examinar transiciones, en este caso de los estudios postobligatorios a la universidad. Y, más concretamente, la distinción de Hillmert y Jacob (2002) entre abandonar los estudios, seguir por la vía académica o seguir por la vía de la formación profesional. Los autores definen esta última vía como la menos arriesgada, ya que daría lugar a un título de formación profesional superior con menor riesgo de compleción - más corto y menos dificultoso que un título universitario- y que, a la vez, permite también a la larga acceder a los estudios superiores, de manera que el estudiante tendría al menos un título en caso de no conseguir 
terminar la carrera universitaria ${ }^{1}$. El factor de valoración del riesgo de no compleción cobra un papel importante en su modelo.

Sin embargo, la perspectiva originalmente planteada por Breen y Goldthorpe (1997) se ha ido ampliando hasta adquirir tintes más sociológicos. En este sentido, la base de la teoría de la elección racional se convierte en elección racional débil ${ }^{2}$, mientras que los factores institucionales, motivacionales, culturales o de percepción se introducen en los nuevos modelos usados por los investigadores ${ }^{3}$. Respecto al trabajo que aquí se presenta se examinan los factores socieconómicos de la familia y el rendimiento académico del individuo que influyen en la transición a la universidad, así como esclarecer si existe interacción entre ellos.

Para realizar dicho análisis aplicamos un modelo de regresión logística binaria que permite pronosticar que un individuo, después de finalizar la educación postobligatoria y, por tanto, teniendo los requisitos requeridos para acceder a la universidad, decide hacer el tránsito a los estudios superiores a partir de un conjunto de características asociadas a sus competencias académicas y/o a la familia. La modelización de este fenómeno se efectúa adoptando una transformación logit. La adopción de este análisis responde a la necesidad de estimar un fenómeno cualitativo que es el resultado, en este caso, de una elección binaria - transitar o no a la universidad.

A tal efecto, definimos una variable dependiente dicotómica $(Y i)$, que toma el valor 1 , es decir $(Y i=1)$, si el individuo $i$ accede o transita a la universidad, y toma valor 0 si no accede o transita, así como un vector $X i$, de dimensión $k$, que comprende las variables predictoras del comportamiento $Y i$. Las $\beta$ constituyen los coeficientes de regresión de las variables independientes introducidas en el modelo, y es el coeficiente para las posibles interacciones. En términos de probabilidad, tenemos la siguiente función:

$$
P(Y=1)=\frac{e^{x^{\prime} \beta}}{1+e^{\prime \beta}}
$$

1. Por otro lado, el hecho de poseer dicho título también supone una reducción del coste de oportunidad de ir a la universidad por parte de este colectivo, al poder compaginar los estudios universitarios con un trabajo remunerado. De hecho, según un análisis realizado en el año 2015 a los alumnos matriculados en las universidades del área euromediterránea, un $46 \%$ de los que acceden a la universidad mediante un CFGS compaginan sus estudios con un trabajo a tiempo parcial o completo, mientras que en el caso de los estudiantes procedentes del bachillerato el porcentaje solo llega un 19\% (Sintes, 2016).

2. La teoría de la elección racional débil permite definir preferencias subjetivas e incompletas, a la par que huye de la concepción del individuo unimotivacional y egoísta de los modelos más economicistas. Martínez García (2004) identifica el denominador común mínimo de esta perspectiva débil: los individuos toman decisiones orientadas a unos objetivos; lo hacen a partir de una ordenación de preferencias (elemento subjetivo) y teniendo en cuenta unas restricciones (elemento objetivo), y llevan a cabo un cálculo de coste-beneficio con el fin de cumplir sus objetivos en la articulación entre lo subjetivo y lo objetivo.

3. Véase por ejemplo la revisión que Fernández Mellizo-Soto (2015) realiza del estudio de la desigualdad de oportunidades educativas remarcando la ampliación de la perspectiva llevada a cabo por Erikson y Jonsson (1996). 
Con

$$
x^{\prime} \beta=\beta_{1}+\beta_{2} X_{2 i}+\beta_{3} X_{31}+\ldots \beta_{k} X_{k i}+\alpha_{1} X_{2 i} X_{3 i}+\ldots+\alpha_{k} X_{k i} X_{k^{\prime} i}
$$

\section{Datos, variables y técnicas de análisis}

Ante la falta de datos que permitan realizar un trabajo de estas características, en el que se pretende descomponer el efecto de las notas del efecto de la clase social al decidir la trayectoria educativa, se recurre a la Enquesta a la Joventut de Catalunya 20124 (EJC2012), la cual pregunta de forma retrospectiva sobre la trayectoria educativa y laboral de los jóvenes catalanes con edades comprendidas entre los 15 y los 34 años, y recoge también información sobre el nivel académico y la situación y la categoría sociolaboral de los padres.

No obstante, de acuerdo con los objetivos de este trabajo, ha sido preciso hacer una selección de esta población. Por un lado, los que han obtenido el título de bachillerato. De ellos se han seleccionado los que tienen de 18 a 34 años $(n$ ponderada $=1077$ ), ya que 18 es la edad mínima en la que es posible acceder a la universidad. Por otro lado, los que han completado un CFGS pueden haber entrado teniendo el título de bachillerato o bien desde la formación profesional media (CFGM) y de otros accesos complementarios. En esta ocasión, la edad mínima en que se puede haber completado todo el itinerario y estar en posesión del título necesario para acceder a la universidad son los 20 años, y por eso se ha seleccionado la población de 20 a 34 años ( $n$ ponderada $=497$ ).

La variable dependiente seleccionada para examinar los ES es la transición a la educación superior. Se trata de una variable dicotómica, donde el valor 1 significa «sí transitar» y el 0 , «no transitar».

Los factores predictores son el rendimiento y la clase social. Las notas son tomadas como medida del rendimiento previo del individuo. Ante la falta de un indicador más objetivo, en este caso se utilizó la pregunta del cuestionario que pedía a los individuos cuáles eran las notas mayoritarias durante la secundaria, por tanto, se trataba de un registro subjetivo del rendimiento académico ${ }^{5}$. Era una variable ordinal de cuatro categorías que se dicotomizó diferenciando entre individuos con «buenas o muy buenas notas» y estudiantes con «malas o muy malas notas». Como clase social de la familia, se tomaron dos indicadores: la ocupación más alta de los progenitores y el nivel de estudios más alto de los progenitores.

4. Queremos agradecer al Observatori Català de la Joventut, del Departament de Treball, Afers Socials i Família, de la Generalitat de Catalunya, la cesión de las informaciones correspondientes a la Enquesta a la Joventut de Catalunya correspondiente a los años 2007 y 2012, como también el apoyo técnico y de asesoría posterior en relación con estas bases de datos.

5. Torrents (2016) afirma que existe un sesgo por clase social con respecto a la autopercepción de las notas como variable independiente al analizar las transiciones. No obstante, los estudios que utilizan las notas como indicador objetivo presentan los mismos resultados, de manera que el rendimiento del estudiante influye en la transición, pero de forma diferente según la clase social de origen. 
Con respecto a las técnicas de análisis utilizadas, en el primer apartado se exponen los resultados de carácter descriptivo mediante tablas de contingencia bivariadas, y en el segundo se incluyen los análisis más explicativos, los cuales pretenden explorar el efecto neto de las diferentes variables analizadas. Este último ejercicio se lleva a cabo mediante la técnica de la regresión logística binaria ${ }^{6}$. Se realizan los análisis diferenciando entre las dos vías de posible acceso - la académica y la de formación profesional-, especialmente porque en los últimos años ha habido cambios en la estructuración de las vías de entrada, y esto ha llevado a un cierto incremento de la presencia de estudiantes procedentes de CFGS en la universidad, con características y expectativas diferenciadas.

\section{Resultados}

\subsection{Perfil de los jóvenes elegibles para acceder a la universidad}

Iniciamos el análisis mediante una exploración de las personas que han obtenido el título necesario para acceder a la universidad y que, por tanto, se encuentran en disposición de elegir si se matriculan en ella o no. El primer colectivo representa la culminación de la educación secundaria superior a través de la vía académica, mientras que el segundo lo sería a través de la vía de la formación profesional. Para cada colectivo analizamos sus características sociodemográficas, las notas previas y la clase social de origen, tomando como variables el nivel de estudios y la ocupación de los progenitores (ver la tabla 1).

Una primera información que obtenemos de los datos analizados es que, del total de jóvenes con opción de ir a la universidad, casi 7 de cada 10 poseen el título de bachillerato, mientras que el $30 \%$ restante posee un ciclo formativo de grado superior. A partir de aquí, la caracterización de cada colectivo está claramente diferenciada. Entre los de la vía académica, tienen una mayor representación las mujeres (57\%); los jóvenes con buenas o muy buenas notas (8 de cada 10 ); el $53 \%$ son hijos e hijas de directivos, gerentes, técnicos y profesionales, y el $47 \%$ de los progenitores poseen estudios superiores. Mientras que, entre los que cursaron un CFGS, tienen una presencia similar hombres y mujeres; los jóvenes con buenas o muy buenas notas representan un 63,3\%, y los padres con ocupaciones que requieren cualificación media (un 63,4\%) y con estudios hasta primarios (un 41,2\%) son los más representativos. También es destacable la heterogeneidad de este colectivo con respecto a su itinerario educativo previo (procedentes de bachillerato, de CFGM o de otros programas formativos).

\subsection{Los determinantes de acceso a la universidad}

Tal y como explica el modelo de EP y ES de Boudon (1974), la decisión de transitar en este caso a la universidad recibe una influencia directa de la clase

6. Tal y como explica Fernández Mellizo-Soto (2015), este es el modelo no lineal usado más frecuentemente desde la década de 1980 en las estimaciones de las transiciones en las bifurcaciones educativas. 
Tabla 1. Caracterización de los colectivos que obtienen el título de bachillerato y el de CFGS

\begin{tabular}{|c|c|c|c|}
\hline & $\begin{array}{c}\text { Título de } \\
\text { bachillerato }\end{array}$ & $\begin{array}{c}\text { Título de } \\
\text { CFGS }\end{array}$ & \\
\hline & $\%$ & $\%$ & $N$ \\
\hline Total & 68,6 & 31,4 & 1569 \\
\hline \multicolumn{4}{|l|}{$\operatorname{Sexo}[0,066]^{\star *}$} \\
\hline Mujer & $\underline{57}$ & 49,9 & 862 \\
\hline Hombre & 43 & $\underline{50,1}$ & 712 \\
\hline \multicolumn{4}{|l|}{ Notas $[0,201]^{* *}$} \\
\hline Malas o muy malas & 18,2 & $\underline{36,7}$ & 378 \\
\hline Buenas o muy buenas & $\underline{81,8}$ & 63,3 & 1193 \\
\hline \multicolumn{4}{|l|}{ Ocupación más alta de los progenitores $[0,210]^{* *}$} \\
\hline Directivos, gerentes, técnicos y profesionales & $\underline{53,1}$ & 31,2 & 705 \\
\hline Ocupaciones que requieren cualificación media & 41,2 & $\underline{63,4}$ & 735 \\
\hline Ocupaciones elementales & 5,7 & 5,4 & 86 \\
\hline \multicolumn{4}{|c|}{ Nivel de estudios más alto de los progenitores $[0,215]^{\star *}$} \\
\hline Superiores & $\underline{47,2}$ & 27,8 & 645 \\
\hline Secundarios & 30,2 & 31 & 478 \\
\hline Hasta primarios & 22,6 & 41,2 & 446 \\
\hline
\end{tabular}

Nota: ${ }^{\star} p \leq 0,05,{ }^{\star *} p \leq 0,01$ prueba $\chi^{2}$. Entre corchetes $\vee$ Cramer. Los porcentajes subrayados son valores más grandes de 1,96 para los residuos corregidos tipificados.

Fuente: elaboración propia a partir de EJC2012.

social de origen, aunque también de forma indirecta esa está condicionada por el rendimiento previo obtenido de los estudiantes (Carabaña, 2008; Jackson, 2013; Bernardi y Cebolla, 2014).

Para reflejar esta cadena de efectos, veamos en primer lugar la influencia que la clase social ejerce sobre las notas (EP). En concreto, utilizamos como variable independiente el nivel de estudios más elevado de los progenitores y como variable dependiente, la percepción subjetiva sobre las notas predominantes en los estudiantes durante la educación secundaria obligatoria.

Tal y como se muestra, en primer lugar es importante resaltar que la distribución por notas favorece a los estudiantes con título de bachillerato. En segundo lugar, atendiendo a las diferencias según las características de los progenitores, las notas presentan una asociación estadística diferencial con cada una de las variables analizadas. Mientras que para los jóvenes de vía académica las notas se relacionan con el nivel educativo de los progenitores y la dirección de la relación es positiva - a más nivel educativo mayor porcentaje de hijos e hijas con buenas notas-, para los jóvenes de vía profesional sus calificaciones están más relacionadas con la ocupación de sus progenitores en un sentido inverso. Es decir, a mayor categoría profesional de los padres, menor porcentaje de hijos e hijas con buenas notas. Esto puede ser un ejemplo de cómo funciona el efecto de compensación, en el sentido de que las familias con ocupaciones de alta cualificación con hijos que muestran bajo rendimiento, por lo tanto con 
Tabla 2. Notas obtenidas en la secundaria postobligatoria según la ocupación y el nivel de estudios de los progenitores. Estudiantes con título de bachillerato y estudiantes con título de CFGS

\begin{tabular}{|c|c|c|c|c|c|c|c|c|}
\hline & \multicolumn{4}{|c|}{$\begin{array}{l}\text { Población con título } \\
\text { de bachillerato }\end{array}$} & \multicolumn{4}{|c|}{$\begin{array}{l}\text { Población con título } \\
\text { de CFGS }\end{array}$} \\
\hline & \multirow{2}{*}{$\begin{array}{c}\text { Buenas } \\
\text { notas } \\
\%\end{array}$} & \multirow{2}{*}{$\begin{array}{c}\text { Malas } \\
\text { notas } \\
\%\end{array}$} & \multicolumn{2}{|c|}{ Total } & \multirow{2}{*}{$\begin{array}{c}\text { Buenas } \\
\text { notas } \\
\%\end{array}$} & \multirow{2}{*}{$\begin{array}{c}\text { Malas } \\
\text { notas } \\
\%\end{array}$} & \multicolumn{2}{|c|}{ Total } \\
\hline & & & $\%$ & $N$ & & & $\%$ & $N$ \\
\hline \multirow[t]{2}{*}{ Total } & 81,9 & 18,1 & 100 & 1043 & 63,1 & 36,9 & 100 & 493 \\
\hline & \multicolumn{4}{|c|}{$\begin{array}{l}\text { Ocupación más alta } \\
\text { de los progenitores }\end{array}$} & \multicolumn{4}{|c|}{$\begin{array}{c}\text { Ocupación más alta } \\
\text { de los progenitores }[0,180]^{\star \star}\end{array}$} \\
\hline $\begin{array}{l}\text { Directivos, gerentes, técnicos } \\
\text { y profesionales }\end{array}$ & 83,5 & 16,5 & 100 & 556 & 49,7 & $\underline{50,3}$ & 100 & 149 \\
\hline $\begin{array}{l}\text { Ocupaciones que requieren } \\
\text { cualificación media }\end{array}$ & 80,3 & 19,7 & 100 & 426 & $\underline{66,9}$ & 33,1 & 100 & 302 \\
\hline \multirow[t]{2}{*}{ Ocupaciones elementales } & 78,7 & 21,3 & 100 & 61 & 77,8 & 22,2 & 100 & 27 \\
\hline & \multicolumn{4}{|c|}{$\begin{array}{l}\text { Nivel de estudios más alto } \\
\text { de los progenitores }[0,102]^{\star \star}\end{array}$} & \multicolumn{4}{|c|}{$\begin{array}{l}\text { Nivel de estudios más alto } \\
\text { de los progenitores }\end{array}$} \\
\hline Superiores & 85,4 & 14,6 & 100 & 508 & 63,5 & 36,5 & 100 & 137 \\
\hline Secundarios & 80,5 & 19,5 & 100 & 318 & 63 & 37 & 100 & 154 \\
\hline Hasta primarios & 75,6 & 24,4 & 100 & 242 & 62,9 & 36,9 & 100 & 202 \\
\hline
\end{tabular}

Nota: ${ }^{*} p \leq 0,05,{ }^{* *} p \leq 0,01$ prueba $\chi^{2}$. Entre corchetes $\vee$ Cramer. Los porcentajes subrayados son valores más grandes de 1,96 para los residuos corregidos tipificados.

Fuente: elaboración propia a partir de EJC2012.

más probabilidades de fracasar en el itinerario de bachillerato y universidad, les empujan a seguir estudiando mediante un CFGS. Probablemente, esta misma situación de malas notas entre familias con ocupaciones elementales conduce a la salida del joven del sistema educativo, donde solo se mantiene el que obtiene buenas notas durante la secundaria obligatoria. Por otro lado, se observa que los hijos e hijas de padres con ocupaciones elementales, ya sean con título de bachillerato o bien con CFGS, se distribuyen de forma muy similar entre los que obtienen buenas notas (un 80\%) y los que obtienen malas notas (un 20\%), lo que puede estar apuntando que entre los jóvenes de familias con ocupaciones de baja cualificación el indicador para valorar si prosiguen o no los estudios sea fundamentalmente la nota, independientemente de si optan por la vía académica o la profesional.

A continuación, examinamos los ES y, por tanto, cómo funciona la variable dependiente que describe la transición a la universidad entre diferentes clases sociales para los dos colectivos observados. En primer lugar lo hacemos analizando el efecto directo del grupo de origen.

En la tabla 3 se constata cómo la propensión de ir a la universidad es mucho mayor entre los jóvenes con título de bachillerato que con el título de CFGS (un $86,2 \%$ frente a un 53,3\%, respectivamente). Para los estudiantes de la vía académica cuyos padres muestran un mayor nivel de estudios y económico, su 
Tabla 3. Transición a la universidad según la ocupación y el nivel de estudios de los progenitores. Estudiantes con título de bachillerato y estudiantes con título de CFGS

\begin{tabular}{|c|c|c|c|c|c|c|c|c|}
\hline & \multicolumn{4}{|c|}{$\begin{array}{l}\text { Población con título } \\
\text { de bachillerato }\end{array}$} & \multicolumn{4}{|c|}{$\begin{array}{l}\text { Población con título } \\
\text { de CFGS }\end{array}$} \\
\hline & \multicolumn{2}{|c|}{$\begin{array}{c}\text { No } \\
\text { Acceso acceso }\end{array}$} & \multicolumn{2}{|c|}{ Total } & \multicolumn{2}{|c|}{$\begin{array}{c}\text { No } \\
\text { Acceso acceso }\end{array}$} & \multicolumn{2}{|c|}{ Total } \\
\hline & $\%$ & $\%$ & $\%$ & $N$ & $\%$ & $\%$ & $\%$ & $N$ \\
\hline \multirow[t]{2}{*}{ Total } & 86,2 & 13,8 & 100 & 1068 & 53,3 & 46,7 & 100 & 484 \\
\hline & \multicolumn{4}{|c|}{$\begin{array}{l}\text { Ocupación más alta } \\
\text { de los progenitores }[0,170]^{\star *}\end{array}$} & \multicolumn{4}{|c|}{$\begin{array}{l}\text { Ocupación más alta } \\
\text { de los progenitores }\end{array}$} \\
\hline $\begin{array}{l}\text { Directivos, gerentes, técnicos } \\
\text { y profesionales }\end{array}$ & $\underline{91}$ & $\underline{9}$ & 100 & 555 & 53,2 & 46,8 & 100 & 139 \\
\hline $\begin{array}{l}\text { Ocupaciones que requieren } \\
\text { cualificación media }\end{array}$ & 83,6 & $\underline{16,4}$ & 100 & 426 & 55,8 & 44,2 & 100 & 303 \\
\hline \multirow[t]{2}{*}{ Ocupaciones elementales } & 68,3 & $\underline{31,7}$ & 100 & 60 & 37 & 63 & 100 & 27 \\
\hline & \multicolumn{4}{|c|}{$\begin{array}{l}\text { Nivel de estudios más alto } \\
\text { de los progenitores }[0,221]^{\star *}\end{array}$} & \multicolumn{4}{|c|}{$\begin{array}{l}\text { Nivel de estudios más alto } \\
\text { de los progenitores }\end{array}$} \\
\hline Superiores & $\underline{93,5}$ & 6,5 & 100 & 506 & 52,2 & 47,8 & 100 & 134 \\
\hline Secundarios & 83,6 & 16,4 & 100 & 323 & 58 & 42 & 100 & 150 \\
\hline Hasta primarios & 74,5 & $\underline{25,5}$ & 100 & 239 & 50,5 & 49,5 & 100 & 200 \\
\hline
\end{tabular}

Nota: ${ }^{*} p \leq 0,05,{ }^{* \star} p \leq 0,01$ prueba $\chi^{2}$. Entre corchetes $\vee$ Cramer. Los porcentajes subrayados son valores más grandes de 1,96 para los residuos corregidos tipificados.

Fuente: elaboración propia a partir de EJC2012.

transición a la universidad está casi asegurada (sobrepasan el 90\%). Mientras que con progenitores con iguales características, solo algo más de la mitad de los jóvenes de la vía profesional alcanzan la universidad. También resulta interesante destacar que, entre los estudiantes de bachillerato, hay claras diferencias entre clases, es decir, frente al 93,5\% de jóvenes de familias con estudios superiores que acceden a la universidad, solo lo hace el 74,5\% de los jóvenes con estudios hasta primarios. Lo mismo ocurre si nos fijamos en las dos categorías extremas del nivel ocupacional de los progenitores. Sin embargo, entre los jóvenes con título de CFGS no aparecen diferencias significativas en el acceso a la universidad dependiendo del nivel educativo u ocupacional de los padres.

A fin de descomponer los dos efectos y ver si persisten, a continuación analizamos qué ocurre en términos de ES teniendo en cuenta las notas. Esto nos lleva a comprobar, en el caso de los jóvenes catalanes entrevistados, un posible efecto de compensación, según el cual, a iguales condiciones (en términos de un mismo rendimiento académico), los jóvenes toman decisiones distintas a la hora de transitar a la universidad de acuerdo con los estudios y la ocupación de sus padres. Esto se explica por un efecto de interacción entre EP y ES. Es decir, tal y como plantean en su trabajo Bernardi y Cebolla (2014), sobre si el efecto marginal de las notas cambia en función de la clase social de origen a la hora de transitar. 
Figura 2. Acceso a la universidad de población con título de bachillerato, notas obtenidas y nivel de estudios de los padres (\%)

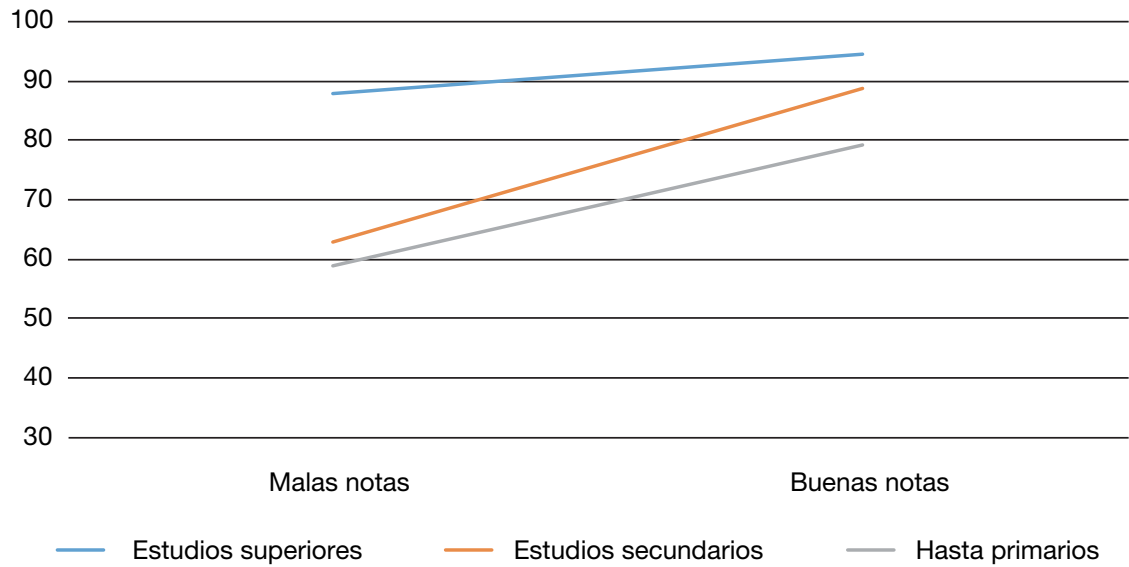

Fuente: elaboración propia a partir de EJC2012.

Figura 3. Acceso a la universidad de la población con título de CFGS, notas obtenidas y nivel de estudios de los padres (\%)

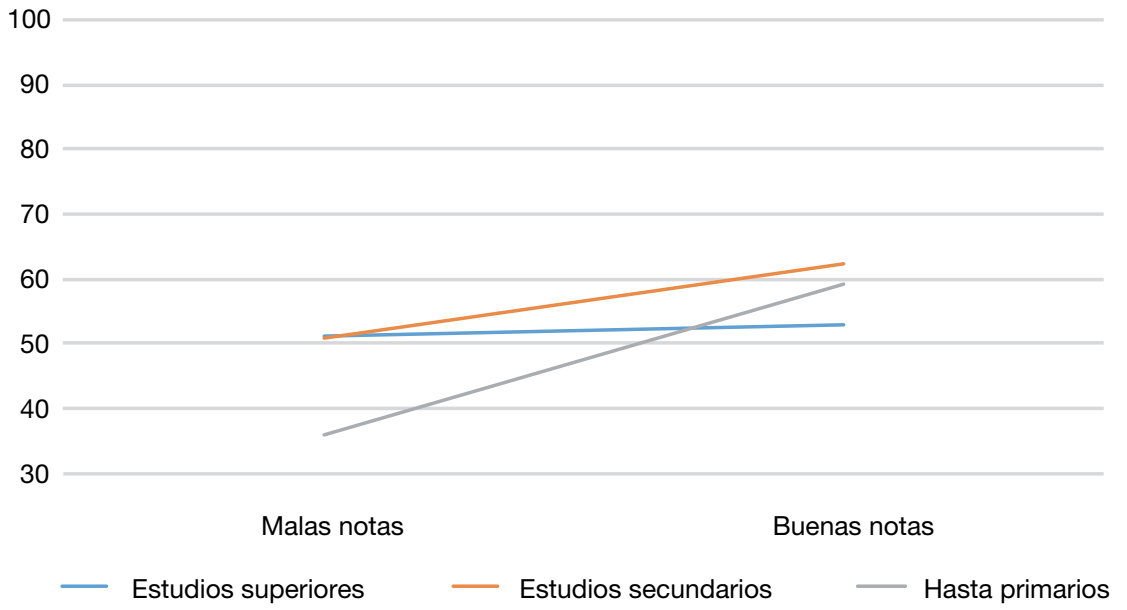

Fuente: elaboración propia a partir de EJC2012.

En este ejercicio se ha optado por la variable que contempla el nivel de estudios de los progenitores como indicador de la clase social familiar para contar con suficiente muestra en los orígenes bajos. El patrón más evidente que se refleja en el gráfico es la mayor propensión a acceder a la universidad por parte de quienes tienen título de bachillerato frente a quienes lo tienen de 
CFGS, tal y como se desprendía de la tabla anterior. Ahora bien, al introducir las notas, lo que se observa claramente es cómo se produce un efecto de compensación entre los que poseen el título de bachillerato. En las familias con estudios superiores la transición está garantizada al margen de las notas que saquen los hijos, mientras que los otros dos grupos son muy dependientes del rendimiento previo de los jóvenes. Es decir: a buenas notas se produce una mayor transición, a malas notas los que transitan son menos.

Aunque en proporciones muy diferentes, una tendencia muy similar se dibuja entre los jóvenes con título de CFGS. De nuevo los hijos de las familias con mayor nivel de estudios son menos dependientes de su rendimiento a la hora de transitar a la universidad. Sean buenos o malos estudiantes, transitan en la misma proporción, aunque en un porcentaje mucho menor (en un 50\% aproximadamente), seguramente porque los que quedan fuera son los estudiantes con peor rendimiento, aquellos de quien las familias no esperan que obtengan ningún otro título académico. En cambio, respecto a las familias con niveles medios y bajos de estudios, las líneas señalan diferentes itinerarios, en el sentido de que los hijos de padres con estudios secundarios tienen una clara propensión a acceder a la universidad al margen de las notas (la línea marca una menor inclinación), mientras que los de origen más bajo dependen fuertemente de las notas obtenidas.

Siguiendo el mismo procedimiento que Bernardi y Cebolla (2014), para contrastar la validez de estos resultados, presentamos los datos del modelo de probabilidad mediante regresión logística en referencia al acceso a la universidad del colectivo de jóvenes con título de bachillerato. Para el colectivo que siguió la vía profesional ${ }^{7}$, como ya se ha podido ver mediante los análisis bivariados, ni el nivel de estudios ni la ocupación de los progenitores resultan significativos a la hora de explicar su transición. Solo ligeramente aparecen las notas como predictor de la trayectoria que siguen después de finalizar un CFGS. Además, la variabilidad de la $Y$ solo alcanza un 3\%.

En la siguiente tabla se presentan los resultados de la estimación de dos modelos logísticos: el primero incluye las variables acerca del nivel de estudios y de la ocupación más elevada de los progenitores, así como las notas obtenidas en la secundaria, y el segundo introduce la interacción de las notas con nivel de estudios y ocupación. Para cada uno de ellos, se incluyen los coeficientes de regresión con sus correspondientes errores estándar (ET), el valor del estadístico de Wald para evaluar la hipótesis nula, la significación estadística asociada y el exponencial de beta.

Como se puede apreciar, el modelo 2 hace aumentar la variabilidad explicada de $Y$ hasta el 17,4\%. Los resultados del modelo 1 muestran el hecho de que obtener buenas notas durante la secundaria y pertenecer a una familia con estudios universitarios son los principales predictores del acceso a la universidad, seguidos de la ocupación de los padres, aunque dicha información muestra menor peso. Al introducir las interacciones entre estas variables (mode-

7. Los resultados se incluyen en el anexo. 
Tabla 4. Análisis de regresión logística binaria por pasos hacia delante. Variable dependiente: transición a la universidad. Población con título de bachillerato

\begin{tabular}{|c|c|c|c|c|c|c|}
\hline & \multicolumn{3}{|c|}{ Modelo 1} & \multicolumn{3}{|c|}{ Modelo 2} \\
\hline & B & Sign. & $\operatorname{Exp}(B)$ & B & Sign. & $\operatorname{Exp}(B)$ \\
\hline \multicolumn{7}{|l|}{ Ocupación de los progenitores } \\
\hline Directivos, gerentes, técnicos y profesionales & 0,98 & ** & 2,664 & $-2,152$ & n.s. & 0,116 \\
\hline Ocupaciones que requieren cualificación media & 0,767 & * & 2,153 & $-1,965$ & n.s. & 0,14 \\
\hline \multicolumn{7}{|l|}{ Ocupaciones elementales } \\
\hline \multicolumn{7}{|l|}{ Nivel formativo de los progenitores } \\
\hline Superiores & 1,206 & ** & 3,34 & 1,746 & ** & 5,733 \\
\hline Secundarios & 0,329 & n. s. & 1,39 & 0,217 & n. s. & 1,242 \\
\hline \multicolumn{7}{|l|}{ Hasta primarios } \\
\hline \multicolumn{7}{|l|}{ Notas } \\
\hline Buenas o muy buenas notas & 1,178 & ** & 3,246 & $-1,996$ & n. s. & 0,136 \\
\hline \multicolumn{7}{|l|}{ Malas o muy malas notas } \\
\hline \multicolumn{7}{|l|}{ Interacciones } \\
\hline Ocupación alta * notas altas & & & & 3,925 & ** & 50,645 \\
\hline Ocupación media * notas altas & & & & 3,323 & ** & 27,754 \\
\hline \multicolumn{7}{|l|}{ Ocupación baja * notas altas } \\
\hline Estudios superiores * notas altas & & & & $-0,824$ & n. s. & 0,439 \\
\hline Estudios secundarios * notas altas & & & & 0,176 & n. s. & 1,193 \\
\hline \multicolumn{7}{|l|}{ Estudios hasta primarios * notas altas } \\
\hline $\mathrm{N}$ & & 1074 & & & 1074 & \\
\hline$\%$ de casos correctamente clasificados & & 85,9 & & & 86,6 & \\
\hline Hosmer \& Lemeshow Test & & 0,379 & & & 0,94 & \\
\hline Nagelkerke $R^{2}$ & & 0,142 & & & 0,174 & \\
\hline
\end{tabular}

Nota: ${ }^{*} p<0,05 ;{ }^{* \star} p<0,01$

Fuente: elaboración propia a partir de EJC2012.

lo 2) sucede lo siguiente: el hecho de tener padres universitarios influye en la decisión de que los hijos accedan a la universidad, independientemente de las notas obtenidas. Sin embargo, en el caso de la ocupación, ocurre que solo las familias con niveles laborales de cualificación alta o media inciden en que los hijos vayan a la universidad cuando han obtenido buenas notas. Dicha circunstancia indica la importancia que parece retomar el factor económico en esta etapa educativa cuando se trata de buenos estudiantes.

Con la finalidad de ver cómo actúan las interacciones de forma más detallada, se analizan los colectivos que han cursado bachillerato y que tienen una probabilidad mayor de acceder a la universidad. En primer lugar, la figura 4 muestra que los jóvenes que acceden a la universidad en un porcentaje mayor son los hijos de familias con estudios universitarios, tal y como muestra la línea discontinua que se ubica en la parte superior del gráfico. Y en segundo lugar, su ligera inclinación evidencia que dicha decisión es independiente de las notas obtenidas durante la secundaria. Por lo tanto, en este caso se trata de una elec- 
Figura 4. Acceso a la universidad de los jóvenes con el título de bachillerato según notas y características familiares

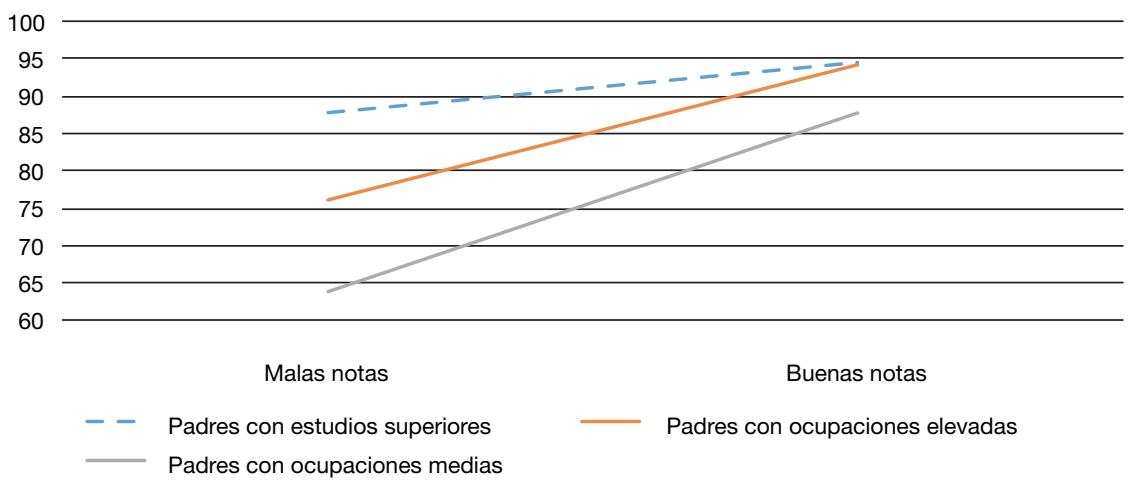

\footnotetext{
* La categoría de nivel ocupacional bajo y notas altas no se muestra aquí, dado que el número de casos obtenidos al cruzar las variables es muy pequeño.
}

Fuente: elaboración propia a partir de EJC2012.

ción muy vinculada al nivel educativo familiar, seguramente como respuesta a las expectativas de los padres y por una cuestión de mantenimiento del estatus y de la clase social de origen, y evitar así una movilidad social descendiente (demotion). Por el contrario, las interacciones relevantes se dan entre el grado ocupacional de los padres, concretamente con los niveles alto y medio, y las calificaciones obtenidas por los hijos. La mayor inclinación de las líneas indica la importancia que tienen las notas de los hijos en estas familias. Si estas son altas, las familias con ocupaciones altas y medias seguramente se arriesgan e invierten sus recursos disponibles para que los hijos vayan a la universidad. Mientras que si las notas de secundaria son malas, ello se relaciona con una menor presencia de jóvenes que acaban matriculándose en estudios superiores. En este caso, las dos líneas se trazan casi en paralelo, puesto que, a mayor nivel ocupacional de los padres, los grados de ingresos son superiores con respecto a los de nivel ocupacional medio, y por tanto disponen de más recursos para invertirlos en la formación de los hijos.

\section{Conclusiones}

Este trabajo quiere contribuir a ampliar el conocimiento sobre las trayectorias académicas seguidas por los jóvenes, en concreto por lo que se refiere a la transición de la educación secundaria postobligatoria a la educación superior y desde la perspectiva de la desigualdad de oportunidades formativas. Somos conscientes de que la investigación realizada parte de una limitación importante que radica en los datos utilizados para medir las notas de los estudiantes durante la secundaria, al tratarse de un indicador subjetivo. Pero es una cuestión que, como apuntan Bernardi y Cebolla (2014), está relacionada con 
la falta sistemática de informaciones recogidas en España para este tipo de investigación educativa, aunque, como novedad, el análisis diferencia entre dos colectivos de estudiantes que hasta ahora han sido tratados en bloque al analizar las desigualdades en el acceso a la universidad.

La conclusión principal de los resultados obtenidos es el comportamiento claramente diferenciado de los dos colectivos analizados: los que están en disposición de acceder a la universidad con el título de bachillerato y los que lo están después de haber cursado un CFGS.

Se ha realizado un análisis de los EP y los ES en ambos colectivos y se ha detectado cómo estos efectos operan de forma diferente en función del título que poseen, pero también de la ocupación y del nivel educativo de los padres.

Respecto al rendimiento (EP), entre los que tienen el título de bachillerato, las notas están claramente relacionadas con el nivel educativo y ocupacional de los progenitores. A mayor nivel, mayor porcentaje de jóvenes con buenas notas. En cambio, no ocurre así con los que poseen el título de CFGS. Este itinerario lo eligen los jóvenes de familias con mayor estatus, o más bien sus padres les empujan a ello, aun cuando sus hijos no muestren tan buen rendimiento.

Respecto al hecho de transitar a la universidad, también se evidencian comportamientos diferenciados según el título que poseen los padres y su nivel educativo. Los hijos de familias con estudios universitarios y con la titulación de bachillerato acceden a la universidad, sean cuales sean las notas obtenidas en la secundaria, lo que nos sitúa ante un claro efecto de compensación en el caso de los jóvenes con el título de bachillerato, dado que allí donde su rendimiento o sus habilidades no son suficientes, las familias les compensan con recursos para alcanzar la universidad. Parece, pues, que ellos tienen ya su itinerario dibujado en razón de su origen social.

En el caso de los jóvenes con el título de CFGS, los niveles de transición son mucho más bajos, aunque entre aquellos procedentes de familias con estudios superiores el acceso es relativamente similar independientemente de su rendimiento.

Muy diferente es el patrón de los hijos de familias con estudios primarios. En este caso, el tránsito a la universidad se relaciona con la obtención de buenas notas en la secundaria, tanto si están en posesión del título de bachillerato como del de CFGS. Dicha circunstancia parece estar señalando que su rendimiento actúa aquí como un claro indicador de su capacidad para superar la siguiente etapa educativa.

Visiblemente diferenciado es el comportamiento que muestran los hijos de familias con estudios secundarios según el título obtenido. En el momento de transitar a la universidad, son claramente dependientes de las notas cuando poseen el título de bachillerato, pero, en cambio, no existen tantas diferencias cuando hablamos de los jóvenes con un CFGS. Así, parece ser que una parte de dicho colectivo emprende este camino más largo con el objetivo de llegar finalmente a realizar estudios superiores, especialmente si sacan buenas notas en esta etapa. 
Finalmente, la ocupación de los padres ha permitido que nos aproximáramos a las cuestiones de carácter más económico y de estatus. Los resultados muestran cómo es una variable que adquiere mucha importancia para explicar el acceso a la universidad para el colectivo que sigue la vía académica, especialmente cuando se trata de estudiantes con un rendimiento previo alto. Teniendo en cuenta el aumento de las tasas universitarias que ha tenido lugar en los últimos años, es evidente que se trata de una decisión en la que las familias con recursos intentan arriesgar lo menos posible. Por lo tanto, puede ser que nos encontremos en un punto de inflexión: si desde la década de 1980 la importancia relativa del coste de los estudios había ido perdiendo peso (Gil Izquierdo et al., 2010), parece ser que ahora vuelve a recuperar protagonismo. El precio de las tasas vuelve a ser importante, y por lo tanto las cuestiones relacionadas con las aspiraciones económicas (aversión relativa al riesgo) se pondrían de manifiesto en esta transición. El resultado no tiene por qué traducirse necesariamente en una renuncia a acceder a la universidad, puesto que, desde un enfoque macro, deben ser analizados otros elementos al mismo tiempo, por ejemplo: qué sucede con la política de becas o con la diferenciación de tasas según territorio y rama de conocimiento, así como qué papel juegan estas variables en el coste de oportunidad calculado por los estudiantes y sus familias. Véanse, si no, las diferentes aportaciones incluidas en el apartado de debate titulado "Los efectos de la crisis sobre el sistema escolar», publicadas en el volumen 27 de la Revista Española de Sociología, donde se discute sobre la relación entre las tasas de escolarización, especialmente la universitaria, y la crisis económica. Desde una perspectiva más micro, antes que la renuncia, se evidencia que han cambiado las pautas de los estudiantes en la elección de estudios, teniendo en cuenta la dificultad y el precio del grado (Troiano et al., 2017). Con lo cual, las conclusiones de este trabajo vienen a complementar estudios recientes como los mencionados y a justificar la necesidad de seguir indagando sobre estas cuestiones.

\section{Referencias bibliográficas}

BECKER, R. y HECKEN, A. E. (2009). «Why are working-class children diverted from universities? -an empirical assessment of the diversion thesis». European Sociological Review, 25 (2), 233-250. <https://doi.org/10.1093/esr/jcn039>

Bernardi, F. (2012). «Unequal Transitions: Selection Bias and the Compensatory Effect of Social Background in Educational Careers». Research in Social Stratification and Mobility, 30 (2), 159-174. <https://doi.org/10.1016/j.rssm.2011.05.005>

BeRnARDi, F. y CeBOlla, H. (2014). «Clase social de origen y rendimiento escolar como predictores de las trayectorias educativas». Revista Española de Investigaciones Sociológicas, 146, 3-22. <https://doi.org/10.5477/cis/reis.146.3>

BERNARDI, F. y REQUENA, M. (2010). «Desigualdad y puntos de inflexión educativos: El caso de la educación post-obligatoria en España». Revista de Educación-MEC, número extraordinario. 
BolIVER, V. (2013). «How fair is access to more prestigious UK Universities?». British Journal of Sociology, 64 (2), 344-364. <https://doi.org/10.1111/1468-4446.12021>

Boudon, R. (1973). L'inégalité des chances: La mobilité sociale dans les sociétés industrielles. París: Armand Colin.

- (1974). Education, opportunity, and social inequality: Changing prospects in western society. New York: John Wiley \& Sons Inc.

BreEN, R. y GOLDTHORPE, J. H. (1997). «Explaining Educational Differentials: Towards a Formal Rational Action Theory». Rationality and Society, 9, 275-305. <https://doi.org/10.1177/104346397009003002>

Breen, R.; RuUd, L.; Walter, M. y PollaK, R. (2009). «Nonpersistent Inequality in Educational Attainment: Evidence from Eight European Countries». American Journal of Sociology, 114, 1475-1521. <https://doi.org/10.1086/595951>

Calero, J.; Choi, A. y Waisgrais, S. (2010). «Determinantes del riesgo de fracaso escolar en España: Una aproximación a través de un análisis logístico multinivel aplicado a PISA-2006». Revista de Educación, número extraordinario, 225-256.

Carabaña Morales, J. y Fuente Blanco, G. de la (2016). «Facultad por Facultad: Origen familiar y empleo de los licenciados en CCSS y Humanidades de la UCM en el año 2003». Revista Complutense de Educación, 27 (3), 983-1001. $<$ https://doi.org/10.5209/rev_rced.2016.v27.n3.47136>

CARABAÑA, J. (2008). Las diferencias entre regiones y paises en las pruebas PISA. Madrid: Colegio Libre de Eméritos. [Publicación electrónica]

- (2015). «La Universidad: Las desigualdades». En: TORRES, C. (ed.). España 2015: Situación social. Madrid: CIS, 565-570.

ERIKSON, R. y JONSSON, J. O. (1996). Can education be equalized?: The Swedish case in comparative perspective. Boulder, Colorado: Westview Press.

Fernández Mellizo-Soto, M. (2015). «Continuidad o cambio en la desigualdad de oportunidades educativas: Evidencia internacional y teorías». Revista Española de Sociología, 23, 151-164.

Gil IzQuierdo, M.; Pablos Escobar, L. de y Martínez Torres, M. (2010). «Los determinantes socioeconómicos de la demanda de educación superior en España y la movilidad educativa intergeneracional». Hacienda Pública Española, 193, 75-108.

HATCHER, R. (1998). «Class Differentiation in Education: Rational choices?». British Journal of Sociology of Education, 19, 5-24. <https://doi.org/10.1080/0142569980190101>

HillmerT, S. y JACOB, M. (2002). «Social inequality in Higher Education: Is vocational training a pathway leading to or away from university?». European Sociological Review, 19 (3), 319-334. <https://doi.org/10.1093/esr/19.3.319>

JaCKSOn, M. (ed.) (2013). Determined to Succeed?: Perfomance versus Choice in educational Attainment. California: Standford University Press.

Marqués-Perales, I. y Gil-HernándeZ, C. J. (2015). «Origen social y sobreeducación en los universitarios españoles: ¿Es meritocrático el acceso a la clase de servicio?». Revista Española de Investigaciones Sociológicas, 150, 89-112. $<$ https://doi.org/10.5477/cis/reis.150.89>

Martínez García, J. S. (2004). «Distintas aproximaciones a la elección racional». Revista Internacional de Sociología, 37, 139-173.

<https://doi.org/10.3989/ris.2004.i37.239> 
MAYER, K.U.; MÜLleR, W. y POLlaK, R. (2007). «Germany: Institutional change and inequalities of access in higher education». En: SHAVIT, Y. (ed.). Stratification in higher education: A comparative study. Stanford, CA: Stanford University Press, 240-265.

OCDE (2013). Panorama de la Educación 2013: España. Nota País.

RAfTerY, A. E. y Hout, M. (1993). «Maximally Maintained Inequality: Expansion, Reform, and Opportunity in Irish Education, 1921-75». Sociology of Education, 66, 41-62. $<$ https://doi.org/10.2307/2112784>

ReAl DeCRETo 558/2010, de 7 de mayo, por el que se modifica el Real Decreto $1892 / 2008$, de 14 de noviembre, por el que se regulan las condiciones para el acceso a las enseñanzas universitarias oficiales de grado y los procedimientos de adminisión a las universidades públicas españolas.

Shavit, Y. y Blossfeld, H.-P. (eds.) (1993). Persistent Inequality: Changing Educational Attainment in Thirteen Countries. Boulder, Colorado: Westview Press.

SINTES, E. (coord.) (2016). Via universitària: Ser estudiant universitari avui. Informe General. Barcelona: Fundació Jaume Bofill i Xarxa Vives d'Universitats.

TORRENTS, D. (2015). «Trayectorias juveniles y factores de la demanda de educación universitaria española para el año 2009», Papers Revista de Sociologia, 100 (1), 131-149. $<$ http://dx.doi.org/10.5565/rev/papers.623>.

- (2016). "La autopercepción de las capacidades: Origen social y elecciones educativas». Revista de la Asociación de Sociología de la Educación (RASE), 9 (1), 78-93.

TRIVENTI, M. (2013). «Stratification in higher education and its relationship with social inequality: A comparative study of 11 European countries». European Sociological Review, 29 (3), 489-502. $<$ https://doi.org/10.1093/esr/jcr092>

Troiano, H.; Torrents, D.; SÁnchez-Gelabert, A. y DazA, L. (2017). «Evolución del acceso a la universidad y de la elección de titulación universitaria entre la población joven en Cataluña». Cuadernos de Relaciones Laborales, 35 (2), 281-303. <https://doi.org/10.5209/crla.56775> 


\section{Anexo}

Tabla A1. Análisis de regresión logística binaria por pasos hacia delante. Variable dependiente: transición a la universidad. Población con título de CFGS

\begin{tabular}{|c|c|c|c|c|c|c|}
\hline & \multicolumn{3}{|c|}{ Modelo 1} & \multicolumn{3}{|c|}{ Modelo 2} \\
\hline & B & Sign. & $\operatorname{Exp}(B)$ & B & Sign. & $\operatorname{Exp}(B)$ \\
\hline \multicolumn{7}{|l|}{ Ocupación de los progenitores } \\
\hline Directivos, gerentes, técnicos y profesionales & 0,861 & n. s. & 2,365 & 1,868 & n. s. & 6,476 \\
\hline Ocupaciones que requieren calificación media & 0,88 & n. s. & 2,411 & 1,278 & n. s. & 3,588 \\
\hline \multicolumn{7}{|l|}{ Ocupaciones elementales } \\
\hline \multicolumn{7}{|l|}{ Nivel formativo de los progenitores } \\
\hline Superiores & $-0,018$ & n. s. & 0,982 & 0,277 & n. s. & 1,319 \\
\hline Secundarios & 0,267 & n. s. & 1,307 & $-0,267$ & n. s. & 0,765 \\
\hline \multicolumn{7}{|l|}{ Hasta primarios } \\
\hline \multicolumn{7}{|l|}{ Notas } \\
\hline Buenas o muy buenas & 0,647 & ** & 1,911 & 1,065 & n. s. & 2,901 \\
\hline \multicolumn{7}{|l|}{ Malas o muy malas } \\
\hline \multicolumn{7}{|l|}{ Interacciones } \\
\hline Ocupación alta * notas altas & & & & $-1,412$ & n. s. & 0,244 \\
\hline Ocupación media * notas altas & & & & $-0,293$ & n. s. & 0,746 \\
\hline \multicolumn{7}{|l|}{ Ocupación baja * notas altas } \\
\hline Estudios superiores * notas altas & & & & 0,900 & n. s. & 1,095 \\
\hline Estudios secundarios * notas altas & & & & 0,532 & n. s. & 1,702 \\
\hline \multicolumn{7}{|l|}{ Estudios hasta primarios * notas altas } \\
\hline$N$ & & 481 & & & 481 & \\
\hline$\%$ de casos correctamente clasificados & & 60,2 & & & 61,1 & \\
\hline Hosmer \& Lemeshow Test & & 0,056 & & & 0,063 & \\
\hline Nagelkerke $R^{2}$ & & 0,048 & & & 0,078 & \\
\hline
\end{tabular}

Nota: ${ }^{\star} p<0,05 ;{ }^{* \star} p<0,01$

Fuente: elaboración propia a partir de EJC2012. 
